\section{Raquianestesia com a Mistura Enantio- mérica de Bupivacaína a 0,5\% Isobárica (S75-R25) em Crianças com Idades de 1 a 5 Anos para Cirurgia Ambulatorial}

\section{Senhor Editor,}

Li com interesse o artigo sobre o emprego de raquianestesia com a mistura enantiomérica de bupivacaína em crianças de 1 a 5 anos para cirurgia ambulatorial ${ }^{1}$, que suscitaram considerações e dúvidas.

Apesar das contribuições importantes dos autores brasileiros, nos anos de 1970, as publicações sobre raquianestesia em crianças, remontam de $1909^{2}$.

Esta técnica popularizou-se à partir dos anos de 1980, como uma alternativa à anestesia geral em recém nascidos e ex-prematuros ${ }^{3-7}$.

Abajian e col. ${ }^{3}$ em 78 recém-nascidos e ex-prematuros, 34 fora do período neonatal, em 81 procedimentos, recomendaram a raquianestesia para crianças. Foram feitas duas tentativas de punções em 73 casos, suplementando com anestesia geral $(n=8)$ e sedação $(n=14)$. Ocorreram 2 bloqueios inadequados e $2 \mathrm{com}$ maior duração que o tempo cirúrgico.

Neste mesmo artigo, a taxa de sucesso, isto é, bloqueio adequado versus procedimento cirúrgico terminado sem nenhuma suplementação, foi de $73 \%$ (59/81).

Sartorelli e col. ${ }^{4}$ em 142 raquianestesias, para procedimentos infraumbilicais, a taxa de sucesso foi de $89 \%$, mas em 38 casos (26,8\%) o sucesso só foi obtido por punção adicional . Outros autores ${ }^{9-15}$ também para procedimentos urológicose de extremidades, relataram as seguintes taxas de sucesso:

Blaise e Roy ${ }^{9}$,em crianças de 7 semanas à 13 anos: punções bem sucedidas, $84 \%(30 / 34)$ raquianestesias únicas $19 / 34(56 \%)$, duração inadequada $(n=5)$; relaxamento muscular fraco $(n=2)$ e falta de condições cirúrgicas $(n=4)$.

Tobias e col. ${ }^{10,11}$ em dois relatos, um recém nascido de 8 dias e outro de 1 dia, bem sucedidos.

Kokki e col. ${ }^{12}$, em 20 crianças de 2 a 5 anos, também bem sucedidos.

Kokki e Hendolin ${ }^{13}, 102$ crianças de 2 meses a 17 anos, $92 \%(90 / 102)$.

Melman e col. ${ }^{14}$, em 100 crianças de 1 mês a 15 anos, $100 \%$.

Parkinson e col. ${ }^{15}$, em 13 crianças de 1 a 7 meses, 99\%.

Rice e col. ${ }^{16}$, em 100 crianças de 1 mês a 1 ano, observaram clinicamente, que o tempo de duração entre injeção e o retorno da flexão do quadril, duravam $56 \pm 2,3$ minutos com lidocaína ( $3 \mathrm{mg} \cdot \mathrm{kg}^{-1}$ ), mais adrenalina $86 \pm 4 \mathrm{mi}-$ nutos; com tetracaína $\left(0,4 \mathrm{mg} \cdot \mathrm{kg}^{-1}\right) 86 \pm 3$ minutos e tetracaína mais adrenalina, $128 \pm 3$ minutos.
Destes 100 pacientes, 87 necessitaram de alguma suplementação adicional inalatória ou venosa, principalmente a cetamina.

Tobias e Mencio ${ }^{17}$ relataram o sucesso obtido através de raquianestesia (tetracaína 0,3-0,6 mg. $\mathrm{kg}^{-1}$ ) para cirurgias de pé torto congênito.

Vários autores ${ }^{3,4,9-18}$, que realizaram raquianestesia em crianças, com idades de 1 dia à 17 anos com drogas hiperbáricas, que foram: tetracaína de $0,2-0,4 \mathrm{mg} \cdot \mathrm{kg}^{-1}$; lidocaína 1,5-3 mg. $\mathrm{kg}^{-1}$; bupivacaína $0,3-0,6 \mathrm{mk} \cdot \mathrm{kg}^{-1}$; dose única de ametocaína de $8 \mathrm{mg}$.

Os anestésicos locais por via raquidiana em crianças diferem dos adultos, o tempo de ação é mais rápido nas crianças menores, mas a duração é curta.

Lidocaína, tetracaína e bupivacaína, sem adrenalina, todos com duração curta clinicamente importante, que não ultrapassaram os 90 minutos $^{19}$.

Em nossa experiência pessoal quando utilizamos tetracaína e bupivacaína, em doses decrescentes, hiperbáricas e com adrenalina, obtivemos também uma duração média de 90 minutos.

Questionamos, no trabalho comentado além das duas falhas (taxa de sucesso de 95\%), ocorreu sedação suplementar, tentativas de punções ou adicionais?

Finalmente alguns conceitos expressados pelo autor, no seu livro "Tratado de Anestesia Raquidiana", me pareceram contraditórios em relação ao artigo comentado, no capítulo "Raquianestesia em Pediatria”, como por exemplo à dose fixa ea indicação ambulatorial ampla ${ }^{20}$.

Atenciosamente.

Carlos Alberto da Silva Júnior, TSA

Rua Esteves Júnior, 545/apto.1202 BI."B"

88015-530 Florianópolis, SC

\section{Spinal Anesthesia for Outpatient Pediatric Surgery in 1 - 5 Years Old Children with $0.5 \%$ Isobaric Enantiomeric Mixture of Bupivacaine (S75-R25)}

Mr. Editor,

I have read with interest the article on spinal anesthesia with $0.5 \%$ isobaric bupivacaine in 1 to 5 -year old children for outpatient procedures ${ }^{1}$, which has raised some considerations and questions.

Although major contributions of Brazilian authors during the 70 s, publications on pediatric spinal anesthesia go back to $1909^{2}$.

This technique has become popular as from 1980 as an alternative to general anesthesia in neonates and former preterm infants ${ }^{3-7}$. 
Abajian et al. ${ }^{3}$ in a study of 81 procedures in 78 neonates and former preterm infants, 34 of them no longer in the neonatal period, recommend spinal anesthesia for children. Two puncture attempts were needed in 73 cases, which were complemented with general anesthesia $(n=8)$ and sedation $(n=14)$. There were 2 inadequate blockades and 2 lasted longer than the surgery.

In this same paper, the success rate, that is adequate blockade versus surgical procedure without complementation, was $73 \%(59 / 81)$.

Sartorelli et al. ${ }^{4}$, in 142 spinal anesthesias for infraumbilical procedures have reported a success rate of $89 \%$, but in 38 cases $(26.8 \%)$ the success was only obtained after additional puncture.

Other authors ${ }^{9-15}$, also for urologic and extremity procedures, have reported the following success rates:

Blaise and Roy $^{9}$ in children aged 7 weeks to 13 years: successful punctures, $84 \%(30 / 34)$, single spinal anesthesias, $56 \%(19 / 34)$, inadequate duration $(n=5)$, poor muscle relaxation $(n=2)$, lack of surgical conditions $(n=4)$.

Tobias et al. ${ }^{10,11}$, two successful reports: an 8-day old and a 1-day old neonate.

Kokki et al. ${ }^{12}$, in 20 children aged 2 to 5 years, also successful.

Kokki and Hendolin ${ }^{13}, 102$ children aged 2 months to 17 years, $92 \%(90 / 102)$

Melman et al. ${ }^{14}, 100$ children aged 1 month to 15 years, $100 \%$.

Parkinson et al. ${ }^{15}$, in 13 children aged 1 to 7 months, $99 \%$.

Rice et al. ${ }^{16}$ have clinically observed, in children aged 1 month to 1 year, that time elapsed between injection and hip flexion return was approximately $56 \pm 2.3$ minutes with lidocaine $\left(3 \mathrm{mg} \cdot \mathrm{kg}^{-1}\right), 86 \pm 4$ minutes with lidocaine plus epinephrine; $86 \pm 3$ minutes with tetracaine $(0.4$ $\left.\mathrm{mg} \cdot \mathrm{kg}^{-1}\right)$, and $128 \pm 3$ minutes with tetracaine plus epinephrine.

From these 100 patients, 87 needed some additional inhalational or venous complementation, especially ketamine.

Tobias and Mencio ${ }^{17}$ have reported the success obtained with spinal anesthesia $\left(0.3-0.6 \mathrm{mg} . \mathrm{kg}^{-1}\right)$ to repair congenital clubfoot.

Several authors ${ }^{3,4,9-18}$ have induced spinal anesthesia in children aged 1 day to 17 years with the following hyperbaric drugs: $0.2-0.4 \mathrm{mg} . \mathrm{kg}^{-1}$ tetracaine; $1.5-3 \mathrm{mg}^{\mathrm{kg}}{ }^{-1}$ lidocaine; 0.3-0.6 mg. $\mathrm{kg}^{-1}$ bupivacaine; $8 \mathrm{mg}$ amethocaine in a single dose.

Spinal local anesthetics behavior in children is different from adults; action time is faster in younger children, but duration is shorter.

Lidocaine, tetracaine and bupivacaine, without epinephrine, have clinically important short duration, not exceeding 90 minutes $^{19}$.

Revista Brasileira de Anestesiologia

Vol. 52, N 5, Setembro - Outubro, 2002
Our personal experience is that with hyperbaric tetracaine and bupivacaine in decreasing doses with epinephrine, mean duration has also been 90 minutes.

We ask: in the study being commented upon, in addition to two failures (success rate of $95 \%$ ), were there sedation complementation, puncture attempts or additional punctures?

Last, but not least, some concepts expressed by the author in his book "Treaty on Spinal Anesthesia", chapter "Spinal Anesthesia in Pediatrics", seem to be contradictory as compared to the article, such as, fixed dose and wide outpatient indication ${ }^{20}$.

Best Regards.

Carlos Alberto da Silva Júnior, TSA,M.D. Rua Esteves Júnior, 545/apto.1202 BI. "B" 88015-530 Florianópolis, SC

\section{REFERÊNCIAS - REFERENCES}

01.Imbelloni LE, Vieira EM, Beato L et al - Raquianestesia com a mistura enantiomérica de bupivacaína a $0,5 \%$ isobárica (S75-R25) em crianças com idades de 1-5 anos para cirurgia ambulatorial. Rev Bras Anestesiol, 2002;52:286-293.

02 . Tyrrel-Gray H - A study of spinal anesthesia in children and infants. Lancet, 1909;2:913-917.

03. Abajian JC, Mellish RWP, Browne AF et al - Spinal anesthesia for surgery in the high-risk infants. Anesth Analg, 1984;63: 359-362.

04. Sartorelli KH, Abajian JC, Kreutz JM et al - Improved outcome utilizing spinal anesthesia in high-risk infants. J Ped Surg, 1992;27:1022-1025.

05. Veverka TJ, Henry DN, Milroy LN et al - Spinal anesthesia reduces the hazard of apnea in high-risk infants. Am Surg, 1991;57:531-534.

06. Harnik EV, Hoy GR, Potolicchio S et al - Spinal anesthesia in premature infants recovering from respiratory distress syndrome. Anesthesiology, 1986;64:95-99.

07. Webster AC, McKishnie JD, Kenyon CF et al - Spinal anaesthesia for inguinal hernia repair in high-risk neonates. Can J Anaesth, 1991;38:281-286.

08. Welborn LG, Rice LJ, Hannallah RS et al - Postoperative apnea in former preterm infants: prospective comparison of spinal and general anesthesia. Anesthesiology, 1990;72: 838-842.

09. Blaise GA, Roy WL - Spinal anaesthesia for minor paediatric surgery. Can Anaesth Soc J, 1986;33:227-230.

10. Tobias JD, Flannagan J, Brock J et al - Neonatal regional anesthesia: alternative to general anesthesia for urologic surgery. Urology, 1993;41:362-365.

11. Tobias JD, Flannagan J - Regional anesthesia in the preterm neonate. Clin Pediatr, 1992;31:668-671.

12. Kokki $\mathrm{H}$, Hendolin $\mathrm{H}$, Vainio $\mathrm{J}$ et al - Operationen im vorschulalter: vergleich von spinalanasthesie und allgemeinanasthesie. Anaesthetist, 1992;41:765-768.

13. Kokki $\mathrm{H}$, Hendolin $\mathrm{H}$ - Comparison of spinal anaesthesia with epidural anaesthesia in paediatric surgery. Acta Anaesthesiol Scand, 1995;39:896-900.

14. Melman E, Penuelas J, Marrufo J - Regional anesthesia in children. Anesth Analg, 1975;54: 387-390. 
15. Parkinson SK, Little WL, Malley RA et al - Use of hyperbaric bupivacaine with epinephrine for spinal anesthesia in infants. Reg Anesth, 1990;15:86-88.

16. Rice LJ, DeMars PD, Whalen TV et al - Duration of spinal anesthesia in infants less than one year of age. Reg Anesth, 1994;19:325-329.

17. Tobias JD, Mencio GA - Regional anesthesia for clubfoot repair in children. Am J Therapeutics, 1998;5:273-277.

18. Hirabayashi Y, Shimizu R, Saitoh K et al - Spread of subarachnoid hyperbaric amethocaine in adolescents. $\mathrm{Br} \mathrm{J}$ Anaesth, 1995;74:41-45

19. Williams RK, Abajian C - Spinal anesthesia in infants. Reg Anesth, 1999;3:170-176.

20. Conceição MJ - Raquianestesia em Pediatria, em Imbelloni LE Tratado de Anestesia Raquidiana. L E Imbelloni, 2001;13: 113-116.

\section{Réplica \\ Raquianestesia com a Mistura Enantio- mérica de Bupivacaína a 0,5\% Isobárica (S75-R25) em Crianças com ldades de 1 a 5 Anos para Cirurgia Ambulatorial}

Senhor Editor,

Agradecemos os comentários de Silva Junior, um dos mais importantes anestesiologistas pediátricos do Brasil, sobre o nosso trabalho ${ }^{1}$. O primeiro relato de raquianestesia em crianças foi, na realidade, de August Bier, em $1899^{2}$, quando a realizou em um menino de 11 anos, utilizando a cocaína isobárica como anestésico. Em 1900, Bainbridge ${ }^{3}$ publicou 40 cirurgias com raquianestesia, incluindo uma criança com menos de três meses de idade. Só em 1909, Tyrell Gray ${ }^{4}$ publicou o resultado de 300 anestesias espinhais em crianças para procedimentos abaixo do diafragma. Vários outros autores relataram o uso da raquianestesia em $1933^{5}, 1936^{6} \mathrm{e}$ $1951^{7}$. Com a introdução dos curares (1944) na prática anestesiológica e o aparecimento de agentes inalatórios mais modernos, como o halotano em 1956, diminuiu o uso da raquianestesia. Por não querermos ser desterrados em nossa terra, preferimos citar autores nacionais quando eles existem, além de serem trabalhos conceituados ${ }^{8,9}$.

A raquianestesia para crianças jamais ganhou popularidade no mundo, até que em 1984 foi reintroduzida como alternativa da anestesia geral para recém-natos de alto risco ${ }^{10-12}$ e se tornou popular para todas as crianças. Nos recém-natos a raquianestesia foi sugerida por diminuir a incidência de complicações, incluindo apnéia e disfunção respiratória no pós-operatório ${ }^{13}$. A anestesia regional diminui a resposta ao estresse cirúrgico durante e após cirurgia, assim como a morbi-mortalidade em pacientes adultos de cirurgias não cardíacas ${ }^{14}$. Os mesmos benefícios foram observados em pacientes pediátricos submetidos à cirurgia cardíaca ${ }^{15}$. Araquianestesia proporciona maior segurança, observando-se menos dessaturação e menor incidência de apnéia ${ }^{11,16-18}$, sendo também indicada em crianças com asma grave, com problemas para intubação traqueal (traqueomalácia, síndrome de Pierre-Robin, artrogripose múltipla congênita, macroglossias etc) e fechamento de ductus arterial. O uso de cateteres no espaço subaracnóideo foi também recentemente proposto ${ }^{19}$. Como se pode apreciar, as indicações têm sido ampliadas durante todo o tempo e, como a anestesia ambulatorial é o grande objetivo do futuro, as indicações foram maiores que na literatura estrangeira. Esses fatos justificam o novo interesse pela técnica, principalmente quando se tem como objetivo estudar uma droga ainda não utilizada em crianças, a mistura enantiomérica ( $\mathrm{S} 75: \mathrm{R} 25)$ de bupivacaína $0,5 \%$ isobárica.

Concordamos com Silva Junior que o comportamento dos anestésicos locais em crianças difere do comportamento nos adultos, sendo o tempo de ação mais rápido, fato observado em nosso trabalho, com latência em torno de 2,29 minutos. Também concordamos que a duração de ação é mais curta, principalmente na anestesia cirúrgica. Entretanto, avaliamos a analgesia residual que ficou em torno de $4 \mathrm{~h} 13$ minutos. Da mesma forma o bloqueio motor desapareceu rapidamente. Admitimos que houve uma falha no trabalho, pois não avaliamos o tempo da cirurgia, mas todos os procedimentos foram realizados com a anestesia proporcionada pela raquianestesia com sedação inalatória com sevoflurano.

Em nosso trabalho não utilizamos o termo taxa de sucesso, porém relatamos a ocorrência de duas falhas e nossa opção por anestesia geral, no lugar de punção adicional. Desta forma, o sucesso de $95 \%$ foi semelhante a diversos trabalhos citados por Silva Junior. Quanto ao questionamento pertinente em relação à sedação suplementar, foi omitido em nosso trabalho, que após a realização da raquianestesia e pesquisa de latência, nível sensitivo e bloqueio motor, foi reintroduzido o agente inalatório sevoflurano sob máscara facial e mantida a hipnose com concentração de $0,5 \%$. Vale salientar que não fez parte do protocolo, mas houve uma economia de 40 a $60 \%$ do custo da anestesia. Este fato é de extrema importância num país como o Brasil.

O capítulo Raquianestesia em Pediatria de meu livro foi escrito por um dos mais conceituados anestesiologistas pediátricos do Brasil, que infelizmente não tem trabalho publicado sobre raquianestesia. Desta forma, o livro mostra a opinião do autor ${ }^{20}$. Certamente, se for reeditado ele poderá reescrever o capítulo citando as novas indicações através de referência brasileira. A verdade tampouco tem história. As coisas são verdadeiras em seu detalhe, se consideradas uma a uma, mas duvidosas em seu conjunto, se consideradas no geral. Da mesma forma, verdades científicas, por mais evidentes e corretas que pareçam em um dado momento, esvaem-se rapidamente frente a novos conceitos e teorias ou novas evidências que modificam seus princípios ${ }^{21}$.

Finalizando, não há verdade científica; há tão somente conhecimentos científicos, que são todos relativos e históricos ${ }^{21}$. Infelizmente não podemos discutir nosso trabalho com a experiência pessoal de Silva Junior, já que ela não está publi- 